\title{
EL USO DE REDES SOCIALES EN LA PRÁCTICA EDUCATIVA DE UNA ASIGNATURA DE POSTGRADO: UNA INVESTIGACIÓN SOBRE EL USO DE LAS TRIC
}

THE USE OF SOCIAL NETWORKS IN EDUCATIONAL PRACTICE IN A GRADUATE DISCIPLINE: AN INVESTIGATION ON THE USE OF ICRTS

Flavio Ferreira Borges y Lucio França Teles
| ferreiraflavioborges@gmail.com | teleslucio@gmail.com |
Universidade de Brasília y Universidade Nacional de Ensino a Distância (UNED), Brasil

Resumen: Este artículo tiene por objetivo investigar el uso de las Tecnologías de la Relación, la Información y la Comunicación (TRIC) en una asignatura del Programa de Postgrado de la Facultad de Educación en Brasil. Las TRIC se utilizaron sistemáticamente en los encuentros de la asignatura, y las redes sociales constituyeron el marco utilizado para las comunicaciones y prácticas pedagógicas. Los encuentros online se realizaron de forma síncrona para que todos los alumnos pudieran comunicarse y relacionarse de forma democrática. El profesor aplicó tres técnicas, las cuales consideró imprescindibles para el buen desarrollo de las actividades: calibración, variabilidad y colaboración. Este estudio presenta los resultados y percepciones sobre el uso de las TRIC en un proceso de enseñanzaaprendizaje que utilizó las tecnologías para promover una educación que realmente pueda marcar la diferencia para nuestros alumnos. La metodología de la investigación realizada en el estudio fue la de investigación-acción, en la cual el profesor utiliza el proceso de enseñanza-aprendizaje como una actividad de investigación, y deja de ser un observador pasivo de la ejecución de las actividades propuestas para asumir una postura de participación, reflexión y de constante modificación en la forma en que actúa. Palabras clave: clases online; educación; prácticas educativas; redes sociales; tecnología. 


\begin{abstract}
This research aims to investigate the use of Information and Communication, Relationship and Technologies (ICRT) in a discipline of the Postgraduate Program of the Faculty of Education in Brazil. The ICRTs were used systematically in the meetings of the discipline and with social networks being the only environment used for communications and pedagogical practices. Online meetings were carried out in a synchronous manner and so that all students could communicate and interact in the virtual environment in a democratic way. The teacher applied three techniques, which he considered essential for the proper development of activities, they are: calibration, variability and collaboration. This study presents the results and perceptions about the use of ICRTs in a teaching-learning process that used technologies to promote an education that can really make a difference for our students. The methodology of the research carried out in the study was that of action research, in which the teacher uses the teaching-learning process as a research activity, ceasing to be a passive observer of the execution of the proposed activities to assume a participation position, reflection and constant change in the way it acts. Keywords: Online Classes; Education; Educational Practices; Social Network; Technology.
\end{abstract}

\title{
1. Introducción: Una práctica educativa con tecnologías digitales
}

Las tecnologías digitales han modificado la difusión y el acceso a la información, y han transformado la relación de las personas con el conocimiento, principalmente las relaciones en la escuela. Barros y Carvalho (2011: 218) colaboran en esta temática diciendo que «las nuevas tecnologías que permiten la interactividad también promueven una nueva relación del alumno con el conocimiento, con otros alumnos y con el profesor».

La posibilidad de que los alumnos interactúen con un fenómeno fuera del aula, antes de que sea presentado por el profesor, se ha convertido en una realidad. Por ejemplo, «nuestros niños consiguen tener acceso a una cantidad de imágenes en un corto espacio de tiempo que el inventor Gutemberg no consiguió en toda su vida» (Gil, 2018: 2). Esto provoca en el profesor la necesidad de modificar la forma en que actúa en el aula, siendo necesario ejercer un papel más como mediador del aprendizaje que como un expositor de contenido.

Enseñar y educar con calidad es el gran desafío de hoy y siempre (Moran, 2006). Según este autor, se da la necesidad de que los profesores sean intelectualmente y emocionalmente curiosos y que sepan motivar y dialogar con estos nuevos alumnos, digitalmente modificados por la sociedad. Por ello, aquel profesor que se propone dar cabida en su aula a lo nuevo, a lo desconocido, que va más allá de su área de actuación y promueva el descubrimiento de 
nuevos contenidos con la participación de los alumnos, promoverá un aprendizaje verdadero. Alerta también Moran (2006) que no es el profesor el único responsable de este cambio, sino que los padres, la administración escolar y los alumnos también deben implicarse en esta nueva educación con calidad, dado que la enseñanza es un proceso social en el que todos están insertos.

El trabajo de Pulita y Santos (2016) resalta la necesidad de cambio en la actuación del profesor en el aula al presentar las desconexiones entre la educación formal y la sociedad, en general, bajo el prisma del uso de las Tecnologías de la Información, la Comunicación y la Expresión (TICE). Por medio de un estudio organizado por categorías, los autores identificaron que hay una desconexión entre la sociedad y las prácticas educativas. Entre esas categorías destacamos dos: la primera identificó que hay un anhelo por parte de los estudiantes en utilizar las tecnologías digitales de su hacer cotidiano en sus actividades educativas; y la otra categoría, destaca que no hay una armonización en la relación entre el uso de las TICE y la escuela, reconociendo que no hay una asociación con el proceso de enseñanza-aprendizaje.

Santos (2014) se manifiesta sobre la desconexión del profesor con la era digital, afirmando que no es que esté excluido del movimiento mediático y tecnológico presente en la sociedad, sino que tiene dificultad para llevar las nuevas tecnologías digitales para sus prácticas en el aula (Mon, Quintero y Segura, 2018). Reconocemos que la escuela no es únicamente el profesor, sino que todos son responsables del éxito del aprendizaje y estamos invitados a promover cambios en la gestión de las instituciones educativas. Asimismo, invitemos a todos los actores del ambiente escolar a proporcionar prácticas que impulsen las relaciones democráticas de difusión del conocimiento (Area, 2010) entre nuestros alumnos. Sin embargo, no olvidemos que el profesor sigue teniendo un papel protagonistas como agente principal en el cambio del comportamiento y el aprendizaje de nuestros alumnos.

En busca de ese entendimiento sobre el papel del profesor en la modernización de los procesos de enseñanza-aprendizaje, la investigación de McKnight et al. (2016) evidencia cómo es fundamental el conocimiento previo de las tecnologías antes de utilizarlas en sistemas escolares tradicionales, con el propósito de transformarlos en ambientes digitales.

El uso de las tecnologías para mediar la comunicación y la interacción «viabiliza la práctica del pensamiento crítico en nuestros alumnos, contribuyendo así con su empoderamiento frente a la sociedad» (Gil Quintana y Marfil-Carmona, 2018: 190). Y ese es el gran desafío, promover acciones que sean reflexivas, críticas y que puedan tener múltiples vías de diálogo entre los participantes, de tal forma que «no sean acciones impuestas por el aparato tecnológico o por el profesor» (Roura-Redondo y Osuna-Acedo, 2018: 105). 
La vida tecnológica cotidiana está repleta de posibilidades, facilidades, formas y medios de relacionarse, y favorece así el acceso a una cantidad de informaciones nunca presenciada por la humanidad. Además, los alumnos poseen una visión de la realidad diferente de los profesores: para ellos «apenas hay distinción entre la vida real y la vida virtual, pues fusionan ambas experiencias como una misma realidad» (Garrido-Lora, Busquet Duran y MuntéRamos, 2016: 52). Esta forma diferente de comprender las tecnologías, entre alumnos y profesores, es conocida como brecha digital y está relacionada con cómo las TICE están siendo utilizadas por los profesores sin explorarse sus prácticas educativas. Es como si existiesen dos mundos: aquel en que todos están conectados en sociedad y el mundo de la escuela lineal, donde las tecnologías no se hacen presentes (Santos, 2014).

Marta-Lazo y Gabelas Barroso (2016) proponen una modificación en la termología utilizada para identificar el uso de las Tecnologías de la Información y la Comunicación (TIC) en actividades educativas, cambiando su sentido a Tecnologías de la Relación, la Información y la Comunicación (TRIC), las cuales tienen como objetivo orientar las prácticas pedagógicas que puedan atenuar la brecha digital, presentada por Garrido-Lora, Busquet Duran y Munté-Ramos, (2016) y las desconexiones de Pulita y Santos (2016) en la utilización de las TICE. La modificación consiste en considerar las relaciones que los alumnos establecen durante la utilización de las tecnologías en su momento de ocio, comprendiendo que esa relación está vinculada a contenidos, formas y contactos seleccionados para promover contenidos significativos para ellos.

La propuesta de la terminología TRIC viene de acuerdo con la necesidad de tener en cuenta que los jóvenes poseen una facilidad y rapidez para seleccionar contenidos de diversas áreas del conocimiento disponibles en Internet, y «más rápido aún para valorarlos y promoverlos en su red de relación, utilizando así sus competencias digitales de forma natural» (Gabelas, Marta-Lazo y González-Aldea, 2015: 5). Este proceso está relacionado con la Intermetodología, que nos permite identificar movimientos de «interacción, interconexión, reciprocidad, recreación e intercompetencia» entre los participantes en el ciberespacio (Marta-Lazo, Marfil-Carmona y Hergueta-Covacho, 2016: 6).

Pasaremos a utilizar el término TRIC por identificar sus principales características en las actividades prácticas intercreativas (Gil Quintana y MarfilCarmona, 2018; Osuna-Acedo y Camarero-Cano, 2016) de este trabajo.

El instrumento utilizado para la construcción de los datos fue la investigación-acción, en la cual el investigador/profesor participó activamente de todas las actividades propuestas para la asignatura objeto de esta investigación. Esta 
actuación fortalece la investigación, una vez que impone al investigador a tener una postura más activa y, con ello, adquiere la compensación en tiempo real para evaluar y reflexionar sobre los resultados obtenidos. Asimismo, propone modificaciones en la forma en que las tareas están siendo ejecutadas con el propósito de hacer el aprendizaje más significativo y actualizado (Latorre, 2005).

\section{Metodología y construcción de los datos}

En esta sección presentaremos la planificación y las etapas de ejecución de las actividades de la asignatura Informática y Comunicación Pedagógica (ICP). La asignatura ICP fue elegida como objeto de estudio por ser ofrecida en un curso de postgrado dirigido a la formación de profesores y por presentar en su plan de enseñanza enfoques prácticos en el uso de tecnologías como medio de comunicación y de expresión pedagógicas para la práctica docente.

En este artículo realizamos el estudio de los datos con el objetivo general de comprender el empoderamiento tecnológico de esos estudiantes bajo unas prácticas educomunicativas. Los objetivos específicos del estudio son:

$\rightarrow$ Realizar prácticas educomunicativas donde tenga un papel central la reflexión crítica sobre el rol de las tecnologías digitales en la comunicación educativa.

$\rightarrow$ Empoderar a los estudiantes y los futuros profesores en la utilización de las TRIC bajo un prisma participativo e implicado con su propio aprendizaje.

La asignatura ICP se impartió en 2018 en el curso de Postgrado en Educación de la Facultad de Educación de la Universidad de Brasilia (Brasil); participaron de la asignatura 17 estudiantes del postgrado y 5 estudiantes de la graduación, que totalizan 22 participantes, entre ellos el investigador de este estudio. En el inicio de las clases el profesor presentó el plan de trabajo y realizó una exposición para anunciar con qué iba a trabajar. Todos los alumnos matriculados en la ICP actuaban en diferentes áreas de la educación, como por ejemplo: prácticas de magisterio, monitoreo en educación primaria, cursos de formación profesional, docencia y administración escolar.

El profesor de ICP se propuso presentar posibles caminos para el uso de dispositivos tecnológicos en el proceso de enseñanza-aprendizaje, y orientó las lecturas en el sentido de incentivar a los alumnos a reconocer propuestas que pudieran promover el éxito en el uso de las TRIC en sus prácticas educativas, principalmente en momentos que extrapolan el aula tradicional. 
Con el propósito de promover la participación e implicación efectiva de los alumnos, el profesor delineó un recorrido metodológico con lecturas de artículos científicos, acompañados de un análisis crítico a partir de la producción de reseñas para fortalecer el conocimiento teórico de los participantes; especialmente en el proceso de mejora de sus habilidades digitales y en la aplicación sistemática de recursos mediáticos, una inquietud latente en la actualidad (Martín y González, 2018). A continuación, presentamos las principales lecturas adoptadas en la asignatura ICP y que tuvieron gran influencia en los asuntos abordados en los encuentros de la asignatura.

El primer artículo leído fue escrito por Edemir José Pulita y Gilberto Lacerda dos Santos (2016) con el título 'As (des)conexões entre a educação e a sociedade: quando e como a escola entrará na era digital?'. Los autores abordan las desconexiones entre la educación formal y la sociedad, en general, bajo el prisma del uso de las TICE. Lo que más llamó la atención de los participantes de la ICP, durante la lectura y discusión, fue el anhelo de los estudiantes por un cambio en la educación a partir de una mayor interacción de las prácticas educativas con las nuevas tecnologías digitales, por una parte, y la preocupación de los profesores con la formación de los niños de corta edad, hoy, involucradas en esta sociedad mediática y tecnológica, sin ningún control o supervisión aparente, por otra.

El artículo 'A promoção da inclusão digital de professores em exercício: uma pesquisa de síntese sobre aproximações entre professores, novas mídias e manifestações culturais emergentes na escola', escrito por Gilberto Lacerda Santos (2014), se presentó con el propósito de abordar la vida cotidiana de los alumnos y cómo esta vida está repleta de nuevas posibilidades y facilidades, cosa que en su gran mayoría no son explotadas por los profesores, comportándose como si existieran dos mundos: aquel en que todos están conectados en sociedad y el mundo de la escuela lineal, donde las tecnologías digitales no se hacen presentes.

En busca del entendimiento sobre el papel del profesor en la modernización de los procesos de enseñanza-aprendizaje, se propuso la lectura del trabajo de McKnight et al. (2016) 'Teaching in a Digital Age: How Educators Use Technology to Improve Student Learning'. En este trabajo los autores presentaron datos sobre el uso de tecnologías en siete ambientes escolares, utilizando entrevistas y grupos focales con los profesores que poseían cierta experiencia en utilizar las TRIC en sus actividades educativas. La investigación presentó la importancia del conocimiento previo de las tecnologías antes de utilizarlas en sistemas escolares, principalmente si el uso es con el propósito de transformarlos en ambientes educativos digitales. 
En la lectura del libro 'Sala de Aula Interactiva', de Marco Silva (2010), se discutía sobre la necesidad de que los educadores se preocupasen por sus prácticas pedagógicas y de implantar nuevas metodologías con el propósito de buscar una sintonía con la era digital y con la construcción de una participación colaborativa entre profesores y alumnos, sea en la educación presencial o en la educación a distancia.

La lectura que tuvo como finalidad provocar un cuestionamiento sobre qué camino estamos recorriendo en la enseñanza fue de los autores Moran, Masetto y Behrens (2000). Los autores convergen para el entendimiento de que es necesario adoptar nuevas prácticas pedagógicas que estén apoyadas en nuevas tecnologías y que puedan promover el conocimiento descentralizado. Para estos autores, el actor principal de ese cambio es el profesor; sin embargo, la simple utilización de los recursos mediáticos para promover la descentralización de la educación no es suficiente, haciéndose necesario modificar la forma de enseñar. Las discusiones llevadas a cabo por los alumnos de ICP, después de la lectura de esta obra, estuvieron relacionadas con cuánto tiempo más necesitaríamos para alcanzar la descentralización del aprendizaje, ya que la obra en cuestión tenía una antigüedad de 18 años y los cambios aún no eran perceptibles.

La reflexión histórica sobre el uso de las tecnologías digitales en actividades educativas quedó a cargo de los autores Kirkorian, Wartella y Anderson (2008). Con el título 'Media and Young Children's Learning', los autores abordan el impacto que los primeros medios provocaron, a lo largo del tiempo, sobre el proceso de aprendizaje de nuestros niños y jóvenes. Además de esa lectura, las discusiones en clase se orientaron también a partir del artículo 'A aprendizagem mediada por TIC: interação e cognição em perspectiva', escrito por Cláudia Maria Arôso Mendes Barbosa (2012), en el que la autora resalta la necesidad de comprender cómo las TIC pueden auxiliar en el proceso de mediación pedagógica, principalmente en Ambientes Virtuales de Aprendizajes (AVA), promoviendo así una aproximación entre alumnos y profesores en la modalidad de Enseñanza a Distancia $(\mathrm{EaD})$ online.

La última lectura propuesta por el profesor de ICP fue escrita por Vani Moreira Kenski (2003) con el título 'Tecnologias e ensino presencial e a distancia'. La discusión orientada a partir de la obra fue sobre la importancia de la interacción social y la comunicación en procesos de enseñanza-aprendizaje, enfatizando que el surgimiento de tecnologías innovadoras no afecta de forma negativa a estas interacciones, sino que refuerza las innovaciones tecnológicas para favorecer la divulgación de las informaciones en una velocidad nunca vista por la humanidad. 
En el curso de las lecturas y debates sobre los temas de los doce textos seleccionados no todos se situaron en esa investigación; el profesor de la asignatura ICP utilizó recursos tradicionales de la clase presencial y de entornos virtuales, siendo la red social Facebook elegida como la principal plataforma virtual. El uso de las redes sociales fue justificado por el profesor por ser un espacio donde los estudiantes tienen confianza para expresar y compartir sus opiniones y, por lo tanto, se convierte en un medio fértil para promover el aprendizaje colaborativo y social, apoyándose en el trabajo de Torres-Diaz, Valdiviezo y Jara (2013: 3).

La primera actividad online propuesta en la ICP fue una transmisión stream en vivo a través de Facebook; sin embargo, la realización de la primera clase virtual síncrona encontró algunas barreras personales ya que, entre los participantes de la asignación ICP, 6 alumnos no poseían perfiles en la referida red social, y el profesor tuvo que negociar con los estudiantes la creación de estos nuevos perfiles. Después de un período de discusión y argumentación entre alumnos y profesores, todos se comprometieron a participar de las actividades online en la referida red social.

Al estudiar individualmente los casos de los participantes que no poseían cuentas en Facebook, se encontró que uno de ellos tenía menos de 28 años y poseía conocimientos en el uso de recursos digitales, por lo que pudo ser considerado como un sabio digital (Prensky, 2012). Preguntado sobre el motivo de no poseer una cuenta en Facebook, el alumno expresó su preocupación por la seguridad y utilización de sus datos personales por parte de la plataforma. El resto de participantes que no poseían perfiles estaban en el grupo de personas que no tenían interés o poca intimidad con redes sociales.

Las actividades comenzaron en la red social Facebook con la creación de un grupo de la asignatura ICP en el perfil del propio profesor, para llevar así a los alumnos al segundo ambiente de aprendizaje, el aula virtual, dado que el primer ambiente estaba en la sala de clase presencial. En ese grupo, el profesor centralizó la mayoría de las prácticas virtuales, y construyó las entradas de las actividades a realizar, noticias, entrega de actividades, divulgación de las notas asignadas a los alumnos y transmisiones en vivo de dos clases.

En los encuentros presenciales, el profesor del curso ICP versaba sobre los contenidos y prácticas presentes en los textos seleccionados y que servía de orientación para las prácticas online. Las prácticas online se realizaron de forma síncrona y, para que todos los alumnos pudieran comunicarse e interactuar de forma democrática en el ambiente virtual, el profesor aplicó las técnicas de calibración, variabilidad y colaboración, que consideró esenciales para el buen desarrollo de las actividades online; estas técnicas se detallan en el cuadro 1 . 
Cuadro 1: Técnicas fundamentales para el éxito de una clase online sincrónica.

\begin{tabular}{|l|l|}
\hline \multicolumn{1}{|c|}{ Técnica } & \multicolumn{1}{c|}{ Justificación de la técnica } \\
\hline $\begin{array}{l}\text { Calibración: establecer intervalos } \\
\text { durante la clase. }\end{array}$ & $\begin{array}{l}\text { Los intervalos son necesarios de modo que sea posible realizar una } \\
\text { calibración en el tiempo de aprendizaje de todos los participantes, ya } \\
\text { que cada alumno posee su tiempo de aprendizaje y la pausa en la clase } \\
\text { proporcionará una alineación en el tiempo de participación de todos. }\end{array}$ \\
\hline $\begin{array}{l}\text { Variabilidad: abordar el contenido } \\
\text { utilizando diferentes técnicas. }\end{array}$ & $\begin{array}{l}\text { Una clase online y en vivo se vuelve más densa en contenido que una clase } \\
\text { presencial; por lo tanto, utilizar diferentes enfoques y recursos se hace } \\
\text { necesario para obtener una participación más efectiva de los alumnos. }\end{array}$ \\
\hline $\begin{array}{l}\text { Colaboración: implicaciones de } \\
\text { los participantes. }\end{array}$ & $\begin{array}{l}\text { Son precisos momentos de participación activa de los alumnos en la clase } \\
\text { online; el profesor debe utilizar los recursos tecnológicos disponibles en } \\
\text { el ambiente con el objetivo de involucrar al alumno en la construcción del } \\
\text { conocimiento, y motivar así una participación colaborativa e intercreativa. }\end{array}$ \\
\hline
\end{tabular}

Fuente: de acuerdo con las orientaciones del profesor de la asignatura ICP.

La técnica 'calibración' tiene por objetivo romper la linealidad de una clase expositiva, ofreciendo a los participantes de la clase online síncrona la posibilidad de revisar el vídeo de parte de la clase, con el propósito de entender mejor algunos conceptos y actividades propuestas. Hay también factores externos al ambiente que puede provocar una ausencia temporal, por ejemplo, fallos en la conexión a Internet o interferencia en el ambiente externo del alumno que causa una ruptura en la concentración del participante y la calibración favorece la reanudación de la atención, necesaria para comprender el contenido que se está presentando.

La utilización de este tipo de recurso interactivo empodera al alumno en su capacidad de comunicación y ofrece al profesor una nueva posibilidad de mediación pedagógica, que extrapola el aula tradicional. Sin embargo, este nuevo modelo de interacción, que puede ser sincrónico y asincrónico, impone a los alumnos una autogestión de su tiempo a dedicarse al aprendizaje y a los profesores una nueva forma de comunicación, más rica y socialmente compartida (Arôso Mendes Barbosa, 2012).

La aplicación de la técnica 'variabilidad' abarca los principios de las TRIC, al estimular al alumno a ejercitar sus competencias digitales, una vez que su participación en la clase online ocurre apoyada en diferentes tecnologías (editor de imagen, buscadores, smartphones) y materiales en variados formatos (vídeos, imágenes, textos, hipervínculos) y, después de la selección y/o producción de los materiales, el alumno debe presentar nuevos significados y construir conjuntamente conocimiento con los demás participantes (Aparici y Osuna-Acedo, 2013).

La técnica 'colaboración' está relacionada con la posibilidad de participación activa de todos los alumnos en la dinámica de una clase online, ya sea por 
medio de foros de discusión, posts de textos, vídeos o imágenes. Este nuevo comportamiento, que la enseñanza online atribuye a sus participantes (OsunaAcedo, Marta-Lazo y Frau-Meigs, 2018), conduce a una práctica educativa basada en los conceptos presentes en las TRIC.

La construcción de los datos ocurrió durante la ejecución de las actividades online y apoyada en la taxonomía de los 10T's, propuesta por OsunaAcedo, Marta-Lazo y Frau-Meigs (2018), que tiene como objetivo orientar acciones que puedan favorecer la transferencia del aprendizaje para obtener éxito en la adquisición del conocimiento. Las diez dimensiones educomunicativas propuestas por las autoras son: tareas auténticas, transferencia del aprendizaje hacia la profesión, transformación pedagógica, TRIC, transmedialidad, temporalidad abierta, transnacionalismo, talento intercreativo, trabajo colaborativo y tolerancia.

\section{Análisis de datos y discusión}

En esta sección discutiremos sobre los datos que fueron construidos a partir de la participación de este investigador y de los demás alumnos en las actividades online propuestas por el profesor de ICP. Aunque la disciplina tenía una propuesta de abordaje práctico solamente el 17,64 por ciento de los encuentros fueron prácticos, es decir, de los diecisiete encuentros tres fueron efectivas prácticas pedagógicas con la utilización de las TRIC. Hubo tres clases online, todas ellas realizadas de forma sincrónica, que utilizaron las redes sociales Facebook y WhatsApp para mediar la comunicación entre los professores y los alumnos.

La primera clase fue transmitida en vivo en el grupo de Facebook; el tema principal de la clase fue el uso de las TRIC y su impacto en la educación. El profesor inició la clase con una orientación sobre las dos pausas de 20 minutos (cada 50 minutos) para la calibración, e invitó a los participantes a contribuir en tiempo real con la clase. De los 22 alumnos matriculados en la asignatura solamente 15 participaron activamente de la actividad en tiempo real. Es posible identificar, a partir de la lista de perfiles activos en el grupo online, que los ausentes eran del grupo de alumnos que no poseían perfiles en Facebook, los mismos que se habían comprometido con el profesor en participar de las clases en la red social, lo que efectivamente no ocurrió.

Los alumnos participantes de la primera classe contribuyeron con 108 posts, un promedio de siete posts por participante. Sin embargo, no todas las contribuciones estaban relacionadas con el contenido de la asignatura. Se seleccionaron para su análisis los comentarios que poseían en su contenido la intención de promover el intercambio de informaciones y que estuvieran alineadas con el tema presentado por el profesor. El cuadro 2 presenta estos posts en orden cronológico en que fueron publicados. 
Cuadro 2: Posts publicados durante la transmisión de la primera clase.

\begin{tabular}{|c|c|}
\hline $\begin{array}{l}\text { Tiempo } \\
\text { (mm:ss) }\end{array}$ & Los comentarios de los participantes de la clase online, según cronología de tiempo \\
\hline 00:00 & La escuela formal es una invención del hombre, Foucault... \\
\hline 01:22 & ¡Vamos a mover gente! \\
\hline $11: 36$ & La dialéctica de la docencia: ¿problema y solución? \\
\hline $24: 13$ & En ese sentido, la autenticidad o incluso autoría y autorización y cuestionable y frágiles. \\
\hline $25: 55$ & Y que el espacio formal es el único valorado por la escuela tradicional. \\
\hline $27: 29$ & Lévy llama «veneno y remedio de la cibercultura». \\
\hline 39:44 & $\begin{array}{l}\text { Las tecnologías deben ser utilizadas para liberar personas y, nunca, para aprisionarlas a máquinas y } \\
\text { conceptos superados. }\end{array}$ \\
\hline 40:55 & Con el uso continuo de las tecnologías tenemos nuevas habilidades humanas y la pérdida de otras. \\
\hline $41: 20$ & $\begin{array}{l}\text { Al mismo tiempo, otras habilidades se estimulan. Por ejemplo, en el trato con los hiper's (texto, } \\
\text { medios de comunicación, links). }\end{array}$ \\
\hline $43: 32$ & $\begin{array}{l}\text { Hay un libro también muy interesante, de Paola Sibilia -'Redes o Paredes'- la escuela en tiempos } \\
\text { de dispersión. }\end{array}$ \\
\hline 44:57 & $\begin{array}{l}\text { ¿Y los profesores nativos digitales? El problema se encuentra en la lucha en traer una nueva cara a } \\
\text { la escuela. }\end{array}$ \\
\hline 46:22 & Un libro interesante es la Educación en Red, una visión emancipadora de la autora Margarita Gómez. \\
\hline $47: 57$ & Los profesores y las computadoras pueden convivir en armonía, por la buena educación. \\
\hline 49:30 & El exceso de información dificulta el filtrado y la selección de contenidos. \\
\hline $58: 37$ & ¡La interacción es necesaria! \\
\hline
\end{tabular}

Fuente: Grupo de la asignatura ICP en Facebook, con la omisión de los nombres de los participantes.

Conforme se ha presentado en el cuadro 2, solamente 15 posturas tuvieron sintonía con el contenido propuesto por el profesor, lo que representa aproximadamente el 14 por ciento del total. Aunque la participación puede ser considerada por debajo de lo esperado, las contribuciones hechas presentaron en sus contenidos un conocimiento previo del asunto por parte de los alumnos y el interés en compartir y contribuir con el aprendizaje de los demás.

Entre los posts es posible notar mensajes de incentivo por una participación activa como, por ejemplo, «iVamos a mover gente!». Hay también mensajes de posicionamiento crítico sobre el tema —en el minuto 44:57—y sugerencias de autores que puedan contribuir con la temática, como Pierre 
Lévy. Observando estas participaciones, es posible considerar que hubo un aprendizaje colaborativo online (Teles, 2015) entre los participantes.

La segunda parte de la primera clase, iniciada después de la primera calibración, fue utilizada para estimular a los participantes a ejercitar sus competencias digitales, aplicando la técnica variabilidad, donde el alumno debería contribuir con el tema a partir del envío de una charges o imagen que contribuyese con la temática de la clase. En esta actividad hubo diez posturas de charges, las cuales tuvieron de uno a dos comentarios o ningún comentario, lo que muestra que el ritmo y la participación de los alumnos disminuyeron. A pesar de la poca participación, este modelo estimula al alumno a dar nuevos significados a contenidos preexistentes, ejercitando así los principios de las TRIC.

La técnica colaboración aplicada en la última parte de la clase está relacionada con la posibilidad de participación activa de los alumnos en la construcción del conocimiento y, como actividad práctica, los participantes tuvieron que construir un pequeño texto para resignificar las imágenes y charges publicados por los participantes en la segunda parte de la clase. Este tipo de propuesta invita a los alumnos a ser activos en el proceso de enseñanza-aprendizaje, lo que exigió una mayor concentración por parte de todos los participantes de la dinámica.

La primera clase online fue finalizada con la participación efectiva de diez alumnos y las contribuciones fueron restringidas al mínimo determinado por el profesor, sin nuevas interacciones relevantes. Se notó que el ritmo de contribuciones fue decayendo conforme el tiempo de la clase iba pasando, lo que tiene su lógica teniendo en cuenta que la clase tuvo una duración de cuatro horas.

La segunda clase online, transmitida por Facebook, presentó una dinámica diferente de la primera clase; el profesor de ICP utilizó tres espacios físicos durante las transmisiones: la oficina de su casa, un parque y su despacho en la universidad. El profesor cambió de espacio físico durante las ejecuciones de las actividades asignadas a los alumnos.

En esta clase, durante los intervalos y locomoción del profesor, los participantes fueron orientados a participar en una discusión en el canal en YouTube (vídeo blog) del profesor Marcos Silva (https://www.youtube.com/ watch?v=EGfw73ZJI4U). De esta forma, se consigue expandir la clase online a otra plataforma e involucrar a otros especialistas del área, lo que posibilita nuevas experiencias para los alumnos y aplica los conceptos presentados en el cuadro 1 .

Al utilizar la dinámica de la movilidad, tanto local como virtual, el profesor evidenció que el espacio físico dejó de ser un limitador para las acciones educativas a quienes se disponen a utilizar las tecnologías como soporte para 
el proceso de enseñanza-aprendizaje, pero no fue posible realizar interacciones e intercambio de experiencias con participantes externos a la clase, pues no hubo retorno de los posts realizados por los alumnos.

La tercera y última clase online fue realizada por WhatsApp, con la creación de un grupo de discusión con los alumnos de la asignatura ICP. La dinámica ocurrió conforme a las orientaciones de las prácticas aplicadas en las clases en Facebook, pero hubo un esfuerzo mayor por parte del profesor en la orientación y gestión del medio. Entre otros aspectos, surgió la preocupación en mantener los posts de forma ordenada y coherente con la secuencia de discusión del contenido y, por eso, las interacciones en esa red social se centraron en el profesor. A pesar de algunas interrupciones y ajustes en el transcurso de las actividades, propuestas en el Whatsapp, los alumnos participantes consideraron el resultado satisfactorio y con la misma densidad en el intercambio de contenidos promovido en las clases anteriores.

En el cierre de la disciplina los alumnos fueron invitados a crear un proyecto para la implementación de una escuela innovadora que utilizara el mayor número posible de recursos tecnológicos en su proceso de enseñanza-aprendizaje. Los proyectos se presentaron en encuentros presenciales y en entornos virtuales.

Los proyectos ficticios, en su gran mayoría, fueron divididos en dos áreas de actuación. Hubo quienes apostaban por una mejora en la infraestructura tecnológica del espacio escolar y propuestas que valoraban más el empoderamiento de los alumnos y profesores a partir de la utilización de tecnologías para mediar en el proceso enseñanza y en el aprendizaje. A pesar de la creatividad en la presentación de las propuestas, no hubo gran distanciamiento de los teóricos leídos y de las prácticas presentadas en ICP.

\section{Conclusiones}

La asignatura ICP que se estudia en esta investigación presentó los desafíos de la mediación pedagógica mediada por las TRIC, promoviendo prácticas online que motivaron la participación activa de los alumnos y el intercambio de informaciones y experiencias entre todos los participantes.

El profesor de ICP utilizó las tecnologías con competencia, demostrando que poseía dominio en las herramientas seleccionadas y que se apoyaba en investigadores y especialistas que reconocen la importancia del uso de diferentes tecnologías digitales en el fortalecimiento del papel del profesor y en el proceso de modernización de la educación. Esto nos lleva a reflexionar acerca de que, lejos de llevar a cabo prácticas educomunicativas, se instauró un uso más tecnocrático de las tecnologías digitales restando protagonismo a los propios sujetos implicados en el proceso de enseñanza-aprendizaje. 
Por ello, al ser una asignatura dirigida a profesionales que actúan en educación y por estar investigando sobre la utilización de tecnologías en sus prácticas pedagógicas, no fue posible identificar entre los participantes una satisfacción con las posibilidades del uso de las tecnologías y la necesidad de empoderarse, lo que corrobora el hecho de la disminución del ritmo en la participación online a partir de la segunda clase y la falta de continuidad de las discusiones en horario extraclase.

Otras dificultades identificadas en los discursos y posts de los alumnos fueron: la interferencia del Estado y la invisibilidad en el proceso educativo. Ellos entienden que las políticas de Estado restringen las prácticas del docente. Así lo podemos comprobar en el comentario en el tiempo 25:55 del cuadro 2. Y la dificultad de reconocerse como posibles agentes transformadores de la educación puede deberse a que todavía no ha surgido el profesor capaz de utilizar las tecnologías de forma eficaz en la práctica educativa, conforme a los comentarios expuestos en los tiempos 44:57 y 47:57 del cuadro 2 .

El uso sistemático de recursos tecnológicos en las prácticas educativas, principalmente en el apoyo al proceso de enseñanza-aprendizaje, aún no es una realidad en el uso cotidiano de los alumnos de la asignatura investigada. A pesar de las propuestas presentadas como posibles alternativas para la utilización de las TRIC de forma efectiva, se seguía observando la presencia arraigada de algunos conceptos relativos a la brecha digital (Garrido-Lora, Busquet Duran y Munté-Ramos, 2016), en la que los alumnos — que son profesores en su mayoría - no tienen un dominio homogéneo de las tecnologías, principalmente en momentos fuera del aula presencial.

Debemos reconocer que es una larga y dura caminata hasta llegar a un ambiente educomunicativo que sea tecnológico y, al mismo tiempo, un vehículo mediador entre la adquisición del conocimiento y la verdadera interacción alumno/profesor. En esto se basarán las futuras líneas de investigación. Cambiar las prácticas docentes no es fácil y encontrar una concepción educomunicativa de la tecnología que venga a dialogar con los saberes acumulados a lo largo de los años se convierte en un ejercicio arduo para los profesores y discípulos de la vieja escuela. 


\section{Referencias bibliográficas}

Aparici, R. y Osuna Acedo, S. (2013). La cultura de la participación. Revista Mediterránea de Comunicación, 4(2), 137-148. Doi: https://doi.org/10.14198/ MEDCOM2013.4.2.07

ArEA, M. (2010). Las redes sociales en Internet como espacios para la formación del profesorado. Razón y Palabra, (63), 1-12.

Arôso Mendes Barbosa, C. M. (2012). A aprendizagem mediada por TIC: interação e cognição em perspectiva. Revista Brasileira de Aprendizagem Aberta e a Distância, 11, 83-100.

Barros, M. das G. y Carvalho, A. B. J. (2011). As concepções de Interatividade nos Ambientes Virtuais de Aprendizagem. En R. P. de Sousa, F. da M. C. da S. C. Moita, y A. B. G. Carvalhoi (Orgs.), Tecnologias digitais na educação (p. 24). Campina Grande: EDUEPB.

Gabelas, J. A.; Marta-Lazo, C. y González-Aldea, P. (2015). El factor relacional en la convergencia mediática: una propuesta emergente. Anàlisi. Quaderns de Comunicació i Cultura, 53, 20-34. Doi: http://dx.doi.org/10.7238/a.v0i53.2509

Garrido-Lora, M.; Busquet Duran, J. y Munté-Ramos, R. À. (2016). De las TIC a las TRIC. Estudio sobre el uso de las TIC y la brecha digital entre adultos y adolescentes en España. Anàlisi. Quaderns de Comunicació i Cultura, 54, 44-57. Doi: http://dx.doi.org/10.7238/a.v0i54.2953

GIL, J. (2018). Interconectados apostando por la construcción colectiva del conocimiento aprendizaje móvil en educación infantil y primaria. Píxel-Bit. Revista de Medios y Educación, 1-15. Doi: http://dx.doi.org/10.12795/pixelbit.2018

Gil Quintana, J. y MarfiL-CARmona, R. (2018). El empoderamiento del alumnado a través de las TRIC. Creaciones narrativas a través de stop motion en educación primaria. index.comunicación, 8(2), 189-210.

Kenski, V. M. (2003). Tecnologias e Ensino Presencial e a Distância. Campinas/ SP: Papirus.

Kirkorian, H. L.; Wartella, E. A. y Anderson, D. R. (2008). Media and Young Children's Learning. The Future of Children, 18(1), 39-61. Doi: https:/ldoi. org/10.1353/foc. 0.0002

LATORRe, A. (2005). La investigación-acción: Conocer y cambiar la práctica educativa $\left(3^{\mathrm{a}}\right)$. Barcelona: Editorial Graó.

Marta-Lazo, C. y Gabelas, J. A. (2016). Comunicación Digital. Un modelo basado en el Factor R-elacional ( $1^{\circ}$ ed). Barcelona: Editorial UOC.

Marta-Lazo, C.; Marfil-Carmona, R. y Hergueta-Covacho, E. (2016). Aplicación de las redes sociales en el aprendizaje en conectividad: Uso del factor relacional en la dialéctica Twitter.Etic@.net, 16(1), 304-319. Recuperado desde: https://goo.gl/hpgyfY 
Martín, A. G. y González, A. T. (2018). Educación Mediática y su Didáctica. Una Propuesta para la Formación del Profesorado en TIC y Medios. Revista Interuniversitaria de Formación del Profesorado, 91(32.1), 15-27.

McKnight, K.; O’Malley, K.; Ruzic, R.; Horsley, M. K.; Franey, J. J.y Bassett, K. (2016). Teaching in a Digital Age: How Educators Use Technology to Improve Student Learning. Journal of Research on Technology in Education, 48(3), 194-211.

Mon, F. M. E.; Quintero, L. J. C. y Segura, J. A. (2018). Un modelo holístico de competencia docente para el mundo digital. Revista Interuniversitaria de Formación del Profesorado, 91, 105-106.

Moran, J. M. (2006). Ensino e aprendizagem inovadores com tecnologias audiovisuais e telemáticas. En J. M. Moran; M. T. Masetto y M. A. Behrens (Orgs.), Novas Tecnologias e Mediação Pedagógica $\left(10^{\mathrm{a}}\right)$. Campinas/SP: Papirus.

Moran, J. M.; Masetto, M. T. y Behrens, M. A. (2000). Novas tecnologias e mediação pedagógica $\left(10^{\mathrm{a}}\right)$. Campinas/SP: Papirus.

Osuna-Acedo, S. y CAmarero-CAno, L. (2016). The ECO european project: A new MOOC dimension based on an intercreativity environment. Turkish Online Journal of Educational Technology, 15(1), 117-125. Doi: https://doi.org/10.1109/TSG.2014.2345886

Osuna-Acedo, S.; Marta-Lazo, C. y Frau-Meigs, D. (2018). From sMOOC to tMOOC, learning towards professional transference. ECO European Project. Comunicar, 26(55). Doi: https://doi.org/10.3916/C55-2018-10

Prensky, M. R. (2012). From digital natives to digital wisdom: Hopeful essays for 21 st century learning. California: Corwin Press.

Pulita, E. J. y Santos, G. L. dos (2016). As (des)conexões entre a educação e a sociedade: quando e como a escola entrará na era digital? Revista Tecnologias na Educação, 17, 11.

Roura-Redondo, M. y Osuna-Acedo, S. (2018). Aprender con el diálogo, dialogar para aprender: Estudio de caso en MOOC. index.comunicación, 8(2), 103-128.

SANTos, G. L. dos. (2014). A promoção da inclusão digital de professores em exercício: uma pesquisa de síntese sobre aproximações entre professores, novas mídias e manifestações culturais emergentes na escola. Revista Inter Ação, 39(3), 529-543. Doi: https://doi.org/10.5216/ia.v39i3.28790

Silva, M. (2010). Sala de aula interativa (5ª, Vol. 1). Rio de Janeiro: Edições Loyola.

Teles, L. (2015). Dimensões da aprendizagem colaborativa no design e gerenciamento de ambientes online. Artefactum. Revista de estudos em linguagem e tecnologia, 11(2), 1-19. 
El uso de redes sociales en la práctica educativa de una asignatura... | Ferreira y Franca | 125

Torres-Diaz, J. C.; Valdiviezo, P. y Jara, D. I. (2013). Integración de redes sociales y entornos virtuales de aprendizaje. Revista de Educación a distancia, 35, 8. Doi: https://doi.org/10.1186/s12905-017-0390-2

Para citar este artículo: Ferreira-Borges, F. y França-Teles, L. (2019). El uso de redes sociales en la práctica educativa de una asignatura de postgrado: una investigación sobre el uso de las TRIC. index.comunicación, 9(1), 109-125. 
